TAIWANESE JOURNAL OF MATHEMATICS

Vol. 18, No. 1, pp. 313-328, February 2014

DOI: $10.11650 /$ tjm.18.2014.3264

This paper is available online at http://journal.taiwanmathsoc.org.tw

\title{
OPERATOR PARAMETERIZATIONS OF $g$-FRAMES
}

\author{
Xunxiang Guo
}

\begin{abstract}
In this paper, we mainly parameterize $g$-frames in Hilbert space in terms of operators. Firstly, under the condition that there exists a $g$-orthonormal basis we establish the one to one correspondence between different kinds of $g$-frames with certain kinds of operators. Then we parameterize $g$-frames without the above restriction. Finally, we consider some special $g$-frames, and their operator parameterizations are established. We also obtain some interesting results on $g$-bases and $g$-dual frames of the transforms by applying the operator parameterizations of $g$-frames.
\end{abstract}

\section{INTRODUCTION}

In 1946, D. Gabor [1] introduced a fundamental approach to signal decomposition in terms of elementary signals. In 1952, Duffin and Schaeffer [2] abstracted Gabor's method to define frames in Hilbert space. Frame was reintroduced by Daubechies, Grossmann, and Meyer [3] in 1986. Today, frame theory is a central tool in many areas such as characterizing function spaces and signal analysis. We refer to [4-10] for an introduction to frame theory and its applications.

Recently, several generalizations of frames in Hilbert spaces have been proposed, in which $g$-frame is one of the most important generalizations which was proposed by Sun in [11]. Sun has pointed out that many other generalizations are special cases of $g$-frames. Let's recall some basic conceptions on $g$-frames first. Let $H$ and $V$ be Hilbert spaces, $\left\{H_{j}: j \in N\right\}$ be a family of closed subspaces of $V . B(H)$ denotes the space of all linear bounded operators from $H$ to $H, B\left(H, H_{j}\right)$ denotes the space of all linear bounded operators from $H$ to $H_{j}$. We call $\left\{\Lambda_{j} \in B\left(H, H_{j}\right): j \in N\right\}$ a $g$-frame for $H$ with respect to $\left\{H_{j}: j \in N\right\}$ if there exist two positive constants $A$ and $B$ such that

$$
A\|f\|^{2} \leq \sum_{j \in N}\left\|\Lambda_{j} f\right\|^{2} \leq B\|f\|^{2}, \forall f \in H,
$$

Received May 1, 2013, accepted August 22, 2013.

Communicated by Ngai-Ching Wong.

2010 Mathematics Subject Classification: 47B35.

Key words and phrases: $g$-Frame, Parameterization, $g$-Basis, $g$-Riesz basis, Operator.

This work was partially supported by SWUFE's Key Subjects Construction Items Funds of 211 Project. 
where $A$ and $B$ are called the lower and upper frame bounds, respectively(for simplification, if the spaces are clear, we will just say that $\left\{\Lambda_{j}: j \in N\right\}$ is a $g$-frame with frame bounds $A$ and $B$ in the sequel). If $A=B$, we call $\left\{\Lambda_{j} \in B\left(H, H_{j}\right): j \in N\right\}$ a g-tight frame. If $A=B=1$, we call $\left\{\Lambda_{j} \in B\left(H, H_{j}\right): j \in N\right\}$ a g-normalized tight frame. If the inequality (1.1) is satisfied only on $\overline{\operatorname{span}}\left\{\Lambda_{j}^{*}\left(H_{j}\right): j \in N\right\}$, then we call $\left\{\Lambda_{j} \in B\left(H, H_{j}\right): j \in N\right\}$ a $g$-frame sequence with respect to $\left\{H_{j}: j \in N\right\}$. $\left\{\Lambda_{j} \in B\left(H, H_{j}\right): j \in N\right\}$ is called $g$-complete with respect to $\left\{H_{j}\right\}$ if $\left\{f: \Lambda_{j} f=\right.$ $0, \forall j\}=\{0\}$. If $\left\{\Lambda_{j} \in B\left(H, H_{j}\right): j \in N\right\}$ is $g$-complete and for any finite subset $J \subset N$ and $f_{i} \in H_{i}, i \in J$, we have

$$
A \sum_{i \in J}\left\|f_{i}\right\|^{2} \leq\left\|\sum_{i \in J} \Lambda_{i}^{*} f_{i}\right\|^{2} \leq B \sum_{i \in J}\left\|f_{i}\right\|^{2},
$$

where $A$ and $B$ are called the lower and upper Riesz bounds, respectively, then we call $\left\{\Lambda_{j} \in B\left(H, H_{j}\right): j \in N\right\}$ a $g$-Riesz basis for $H$ with respect to $\left\{H_{j}: j \in N\right\}$. It is well known that if $\left\{\Lambda_{j} \in B\left(H, H_{j}\right): j \in N\right\}$ is a $g$-frame with respect to $\left\{H_{j}: j \in N\right\}$, then the operator $S: H \rightarrow H$ defined by

$$
S f=\sum_{j \in N} \Lambda_{j}^{*} \Lambda_{j} f
$$

is well defined and it is also an invertible bounded operator on $H$. $S$ is called the frame operator associated with $\left\{\Lambda_{j} \in B\left(H, H_{j}\right): j \in N\right\}$. It is also well known that $\left\{\Lambda_{j} S^{-1}: j \in N\right\}$ is a $g$-frame for $H$ with respect to $\left\{H_{j}: j \in N\right\}$ as well. And $\left\{\Lambda_{j} S^{-1}: j \in N\right\}$ is called the canonical dual g-frame of $\left\{\Lambda_{j}: j \in N\right\}$ and it is denoted by $\left\{\widetilde{\Lambda}_{j}: j \in N\right\}$. All the $g$-frames $\left\{\Gamma_{j}: j \in N\right\}$ which satisfy

$$
f=\sum_{j \in N} \Lambda_{j}^{*} \Gamma_{j} f, \forall f \in H
$$

are called dual $g$-frames of $\left\{\Lambda_{j}: j \in N\right\}$.

$g$-Frames in Hilbert spaces have been studied intensively, for more details see [1220] and the references therein. Recently, there are many mathematicians who apply operator theory tools to do research on frame theory in Hilbert spaces, in particular, they apply operator techniques to consider the construction of frames, which is a basic problem in theory and applications. Many interesting and useful results are obtained, we refer to [21-25] as references for this. In this paper, we apply the operator theory tools to consider the similar problems on $g$-frame theory. In section 2, we introduce the definitions and lemmas which are needed in the sequel. In section 3, given a $g$ orthonormal basis of $H$, we establish the one to one correspondence between all kinds of $g$-frames with certain operators. In section 4, we parameterize the $g$-frames in general. In section 5, some special $g$-frames are considered and their operator parameterizations are obtained. 


\section{Definitions And Lemmas}

In this section, we give the definitions and lemmas which we need in the sequel.

Definition 2.1. For each Hilbert space sequence $\left\{H_{i}\right\}_{i \in N}$, we define the space $l^{2}\left(\oplus H_{i}\right)$ by

$$
l^{2}\left(\oplus H_{i}\right)=\left\{\left\{f_{i}\right\}_{i \in N}: f_{i} \in H_{i}, i \in N, \sum_{i=1}^{+\infty}\left\|f_{i}\right\|^{2}<+\infty\right\} .
$$

With the inner product defined by $\left\langle\left\{f_{i}\right\},\left\{g_{i}\right\}\right\rangle=\sum_{i=1}^{+\infty}\left\langle f_{i}, g_{i}\right\rangle$, it is easy to see that $l^{2}\left(\oplus H_{i}\right)$ is a Hilbert space.

Definition 2.2. $\left\{\Lambda_{j} \in B\left(H, H_{j}\right)\right\}_{j=1}^{\infty}$ is called $g$-linearly independent with respect to $\left\{H_{j}\right\}$ if $\sum_{j=1}^{\infty} \Lambda_{j}^{*} g_{j}=0$, then $g_{j}=0$, where $g_{j} \in H_{j}(j=1,2, \cdots)$.

Definition 2.3. $\left\{\Lambda_{j} \in B\left(H, H_{j}\right)\right\}_{j=1}^{\infty}$ and $\left\{\Gamma_{j} \in B\left(H, H_{j}\right)\right\}_{j=1}^{\infty}$ are called $g$ biorthonormal with respect to $\left\{H_{j}\right\}$, if

$$
\left\langle\Lambda_{j}^{*} g_{j}, \Gamma_{i}^{*} g_{i}\right\rangle=\delta_{j, i}\left\langle g_{j}, g_{i}\right\rangle, \forall j, i \in N, g_{j} \in H_{j}, g_{i} \in H_{i} .
$$

Definition 2.4. We say $\left\{\Lambda_{j} \in B\left(H, H_{j}\right)\right\}_{j=1}^{\infty}$ is a g-orthonormal basis for $H$ with respect to $\left\{H_{j}\right\}$, if it is g-biorthonormal with itself and for any $f \in H$ we have

$$
\sum_{j \in N}\left\|\Lambda_{j} f\right\|^{2}=\|f\|^{2}
$$

Remark 2.5. Equation (2.1) implies that a $g$-orthonormal basis is a $g$-normalized tight frame.

Definition 2.6. We call $\left\{\Lambda_{j} \in B\left(H, H_{j}\right)\right\}_{j=1}^{\infty}$ a g-basis for $H$ with respect to $\left\{H_{j}\right\}$ if for any $x \in H$ there is a unique sequence $\left\{g_{j}\right\}$ with $g_{j} \in H_{j}$ such that

$$
x=\sum_{j=1}^{\infty} \Lambda_{j}^{*} g_{j} .
$$

Remark 2.7. $g$-basis is a natural generalization of Schauder basis, for more details, please see [20].

Definition 2.8. Suppose $\left\{\Lambda_{j}: j \in N\right\}$ is a g-Riesz basis of $H$ with respect to $\left\{H_{j}: j \in N\right\}$ and $\left\{\Gamma_{j}: j \in N\right\}$ is a g-Riesz basis of $Y$ with respect to $\left\{H_{j}: j \in N\right\}$. If there is a homemorphism $S: H \rightarrow Y$ such that $\Lambda_{j}=\Gamma_{j} S$ for each $j \in N$, then we say that $\left\{\Lambda_{j}: j \in N\right\}$ and $\left\{\Gamma_{j}: j \in N\right\}$ are equivalent. 
Lemma 2.9. ([5]). Suppose that $T: K \rightarrow H$ is a bounded surjective operator. Then there exists a bounded operator (called the pseudo-inverse of T) $T^{\dagger}: H \rightarrow K$ for which

$$
T T^{\dagger} f=f, \forall f \in H .
$$

Lemma 2.10. ([20]). If $\left\{\Lambda_{j}: j \in N\right\}$ is a g-basis for $H$ with respect to $\left\{H_{j}\right.$ : $j \in N\}$, then $\left\{\Lambda_{j}: j \in N\right\}$ is g-complete and g-linearly independent with respect to $\left\{H_{j}: j \in N\right\}$.

Lemma 2.11. ([11]). A g-Riesz basis $\left\{\Lambda_{j}: j \in N\right\}$ is an exact g-frame. Moreover, it is g-biorthonormal with respect to its dual $\left\{\widetilde{\Lambda}_{j}: j \in N\right\}$.

Lemma 2.12. A sequence $\left\{\Lambda_{j}: j \in N\right\}$ is a $g$-frame for $H$ if and only if

$$
T:\left\{f_{i}: i \in N\right\} \rightarrow \sum_{i \in N} \Lambda_{i}^{*} f_{i}
$$

is a well-defined and bounded mapping from $l^{2}\left(\oplus H_{j}\right)$ onto $H$.

Lemma 2.13. ([20]). Let $\Lambda_{j} \in B(H, H j), j \in N$. Then the following statements are equivalent:

(1) The sequence $\left\{\Lambda_{j}\right\}_{j \in N}$ is a g-Riesz basis for $H$ with respect to $\left\{H_{j}\right\}_{j \in N}$.

(2) The sequence $\left\{\Lambda_{j}\right\}_{j \in N}$ is a g-frame for $H$ with respect to $\left\{H_{j}\right\}_{j \in N}$ and $\left\{\Lambda_{j}\right\}_{j \in N}$ is g-linearly independent.

(3) The sequence $\left\{\Lambda_{j}\right\}_{j \in N}$ is a g-basis and a g-frame with respect to $\left\{H_{j}\right\}_{j \in N}$.

Lemma 2.14. Every g-frame $\left\{\Lambda_{j}: j \in N\right\}$ is g-complete.

Proof. Since $\left\{\Lambda_{j}: j \in N\right\}$ is a $g$-frame, there exist positive constants $A$ and $B$ such that

$$
A\|f\|^{2} \leq \sum_{j \in N}\left\|\Lambda_{j} f\right\|^{2} \leq B\|f\|^{2}, \forall f \in H
$$

So if $\Lambda_{j} f=0, \forall f \in H$, then $f=0$. It follows that $\left\{\Lambda_{j}: j \in N\right\}$ is $g$-complete.

Lemma 2.15. ([13]). $\left\{\Lambda_{j}: j \in N\right\}$ is $g$-complete if and only if $\overline{\operatorname{span}}\left\{\Lambda_{j}^{*}\left(H_{j}\right)\right.$ : $j \in N\}=H$.

Lemma 2.16. ([11]). A sequence $\left\{\Lambda_{j}: j \in N\right\}$ is a g-Riesz basis for $H$ with respect to $\left\{H_{j}: j \in N\right\}$ if and only if there is a g-orthonormal basis $\left\{\theta_{j}: j \in N\right\}$ for $H$ and a bounded invertible linear operator $T$ on $H$ such that $\Lambda_{j}=\theta_{j} T, j \in N$. 
Lemma 2.17. Suppose that $\left\{\Lambda_{j} \in B\left(H, H_{j}\right): j \in N\right\}$ is g-complete and $T \in$ $B(H)$. Then $\left\{\Lambda_{j} T: j \in N\right\}$ is $g$-complete if and only if $T$ is injective.

Proof. $\Rightarrow$ : Suppose that $\Lambda_{j} T f=0$ for every $j \in N$. Since $\left\{\Lambda_{j}: j \in N\right\}$ is $g$-complete, $T f=0$. Since $T$ is injective, $f=0$. It implies that $\left\{\Lambda_{j} T: j \in N\right\}$ is $g$-complete.

$\Leftarrow$ : Suppose that $T f=0$. Then $\Lambda_{j} T f=0$ for every $j \in N$. Since $\left\{\Lambda_{j} T: j \in N\right\}$ is $g$-complete, $f=0$. It implies that $T$ is injective.

\section{Conditional Operator Parameterizations of $g$-Frames}

In this section, we mainly parameterize the $g$-frames under the assumption that there exists a $g$-orthonormal basis for $H$.

Theorem 3.1. Suppose that $\left\{\theta_{j}: j \in N\right\}$ is a g-orthonormal basis for $H$. Then $\left\{\Lambda_{j}: j \in N\right\}$ is a g-normalized tight frame sequence if and only if there exists a unique partial isometry $T \in B(H)$ such that $\Lambda_{j}=\theta_{j} T, \forall j \in N$.

Proof. $\Rightarrow$ : Suppose that $\left\{\Lambda_{j}: j \in N\right\}$ is a $g$-normalized tight frame sequence. Define a linear operator $T$ by

$$
T x=\sum_{j \in N} \theta_{j}^{*} \Lambda_{j} x, x \in \overline{\operatorname{span}}\left\{\Lambda_{j}^{*}\left(H_{j}\right): j \in N\right\},
$$

and $T x=0, x \in\left(\overline{\operatorname{span}}\left\{\Lambda_{j}^{*}\left(H_{j}\right): j \in N\right\}\right)^{\perp}$. Since for any $x \in \overline{\operatorname{span}}\left\{\Lambda_{j}^{*}\left(H_{j}\right): j \in\right.$ $N\}$, we have

$$
\|T x\|^{2}=\left\|\sum_{j \in N} \theta_{j}^{*} \Lambda_{j} x\right\|^{2}=\sum_{j \in N}\left\|\Lambda_{j} x\right\|^{2}=\|x\|^{2},
$$

$T$ is isometric on $\overline{\operatorname{span}}\left\{\Lambda_{j}^{*}\left(H_{j}\right): j \in N\right\}=(\operatorname{Ker} T)^{\perp}$. So $T$ is a partial isometry. Since $\left\{\theta_{j}: j \in N\right\}$ is a $g$-orthonormal basis, we have

$\left\langle\theta_{j} \theta_{k}^{*} g_{k}, g_{j}\right\rangle=\left\langle\theta_{k}^{*} g_{k}, \theta_{j}^{*} g_{j}\right\rangle=\delta_{j, k}\left\langle g_{k}, g_{j}\right\rangle=\left\langle\delta_{j, k} g_{k}, g_{j}\right\rangle, \forall j, k \in N, g_{j} \in H_{j}, g_{k} \in H_{k}$.

So, for any $j \in N$ and any $x \in \overline{\operatorname{span}}\left\{\Lambda_{j}^{*}\left(H_{j}\right): j \in N\right\}$, we have

$$
\theta_{j} T x=\theta_{j} \sum_{k \in N} \theta_{k}^{*} \Lambda_{k} x=\Lambda_{j} x
$$

If $x \in\left(\overline{\operatorname{span}}\left\{\Lambda_{j}^{*}\left(H_{j}\right): j \in N\right\}\right)^{\perp}$, then $\theta_{j} T x=0$. Since

$$
\left\langle\Lambda_{j} x, g_{j}\right\rangle=\left\langle x, \Lambda_{j}^{*} g_{j}\right\rangle=0, \forall g_{j} \in H_{j}, \forall x \in\left(\overline{\operatorname{span}}\left\{\Lambda_{j}^{*}\left(H_{j}\right): j \in N\right\}\right)^{\perp},
$$


$\Lambda_{j} x=0$ for all $x \in\left(\overline{\operatorname{span}}\left\{\Lambda_{j}^{*}\left(H_{j}\right): j \in N\right\}\right)^{\perp}$. It follows that

$$
\theta_{j} T x=\Lambda_{j} x, \forall x \in H,
$$

i.e., $\theta_{j} T=\Lambda_{j}, \forall j \in N$. If there exist partial isometries $T_{1}, T_{2}$ such that $\theta_{j} T_{1}=$ $\theta_{j} T_{2}=\Lambda_{j} \forall j \in N$, then $T_{1}^{*} \theta_{j}^{*}=T_{2}^{*} \theta_{j}^{*} \forall j \in N$. Hence for any $j \in N$ and $g_{j} \in H_{j}$, we have that $T_{1}^{*} \theta_{j}^{*} g_{j}=T_{2}^{*} \theta_{j}^{*} g_{j}$. But $\overline{\operatorname{span}}\left\{\theta_{j}^{*}\left(H_{j}\right)\right\}=H$, so $T_{1}^{*}=T_{2}^{*}$. It follows that $T_{1}=T_{2}$. So the partial isometry which satisfies the condition is unique.

$\Leftarrow$ : If there exists a partial isometry $T$ such that $\Lambda_{j}=\theta_{j} T, \forall j \in N$, then

$$
\sum_{j \in N}\left\|\Lambda_{j} f\right\|^{2}=\sum_{j \in N}\left\|\theta_{j} T f\right\|^{2}=\|T f\|^{2} .
$$

Since $\|T f\|^{2}=\|f\|^{2}$ for all $f \in(\operatorname{Ker} T)^{\perp}=\operatorname{Range}\left(T^{*}\right)=\overline{\operatorname{span}}\left\{T^{*} \theta_{j}^{*}\left(H_{j}\right): j \in\right.$ $N\}=\overline{\operatorname{span}}\left\{\Lambda_{j}^{*}\left(H_{j}\right): j \in N\right\}$. So $\left\{\Lambda_{j}: j \in N\right\}$ is a $g$-normalized tight frame sequence.

Corollary 3.2. Suppose that $\left\{\theta_{j}: j \in N\right\}$ is a g-orthonormal basis for $H$. Then $\left\{\Lambda_{j}: j \in N\right\}$ is a g-normalized tight frame for $H$ if and only if there exists a unique isometry $T \in B(H)$ such that $\Lambda_{j}=\theta_{j} T, \forall j \in N$.

Proof. If $\left\{\Lambda_{j}: j \in N\right\}$ is a $g$-normalized tight frame for $H$, then there exists a unique partial isometry $T$ such that $\Lambda_{j}=\theta_{j} T, \forall j \in N$ by Theorem 3.1. So it is sufficient to show that $T$ is an isometry. Suppose that $T f=0$. Then

$$
0=\|T f\|^{2}=\sum_{j \in N}\left\|\theta_{j} T f\right\|^{2}=\|f\|^{2} .
$$

So $f=0$. It implies that $\operatorname{Ker} T=\{0\}$. Since $T$ is a partial isometry, $T$ is isometric on $(\operatorname{Ker} T)^{\perp}=H$, which means that $T$ is an isometry. Conversely, if there is an isometry $T$ such that $\Lambda_{j}=\theta_{j} T \forall j \in N$, then $\left\{\Lambda_{j}: j \in N\right\}$ is a $g$-normalized tight frame sequence by Theorem 3.1. So for any $f \in \overline{\operatorname{span}}\left\{\Lambda_{j}^{*}\left(H_{j}\right): j \in N\right\}$, we have that

$$
\|f\|^{2}=\sum_{j \in N}\left\|\Lambda_{j} f\right\|^{2}
$$

To show that $\left\{\Lambda_{j}: j \in N\right\}$ is a $g$-normalized tight frame for $H$, it is sufficient to show that $\overline{\operatorname{span}}\left\{\Lambda_{j}^{*}\left(H_{j}\right): j \in N\right\}=H$. Suppose that $f \perp \overline{\operatorname{span}}\left\{\Lambda_{j}^{*}\left(H_{j}\right): j \in N\right\}$, then

$$
\left\langle f, \Lambda_{j}^{*} g_{j}\right\rangle=\left\langle f, T^{*} \theta_{j}^{*} g_{j}\right\rangle=\left\langle T f, \theta_{j}^{*} g_{j}\right\rangle=0, \forall j \in N, g_{j} \in H_{j} .
$$

Since $\left\{\theta_{j}: j \in N\right\}$ is a $g$-orthonormal basis, it is a $g$-normalized tight frame. So $\left\{\theta_{j}: j \in N\right\}$ is $g$-complete by Lemma 2.14. Hence $\overline{\operatorname{span}}\left\{\theta_{j}^{*}\left(H_{j}\right): j \in N\right\}=H$ by Lemma 2.15. So $T f=0$. Since $T$ is an isometry, $f=0$. It follows that $\overline{\operatorname{span}}\left\{\Lambda_{j}^{*}\left(H_{j}\right): j \in N\right\}=H$. 
Theorem 3.3. Suppose that $\left\{\theta_{j}: j \in N\right\}$ is a g-orthonormal basis for $H$. Then $\left\{\Lambda_{j}: j \in N\right\}$ is a g-frame sequence for $H$ with fame bounds $a$ and $b$ if and only if there exists $a$ unique $T \in B(H)$ such that $\Lambda_{j}=\theta_{j} T, \forall j \in N$ and $a P \leq T T^{*} \leq b P$ for some orthogonal projection.

Proof. The uniqueness of $T$ can be verified similarly as we do in Theorem 3.1. $\Rightarrow$ : Define a linear operator $T$ by

$$
\begin{aligned}
& T x=\sum_{j \in N} \theta_{j}^{*} \Lambda_{j} x, \forall x \in \overline{\operatorname{span}}\left\{\Lambda_{j}^{*}\left(H_{j}\right): j \in N\right\}, \\
& T x=0, \forall x \in\left(\overline{\operatorname{span}}\left\{\Lambda_{j}^{*}\left(H_{j}\right): j \in N\right\}\right)^{\perp} .
\end{aligned}
$$

Then it is easy to verify that

$$
\begin{aligned}
& \theta_{j} T x=\Lambda_{j} x, \forall x \in \overline{\operatorname{span}}\left\{\Lambda_{j}^{*}\left(H_{j}\right): j \in N\right\}, \forall j \in N, \\
& \theta_{j} T x=\Lambda_{j} x=0, \forall x \in\left(\overline{\operatorname{span}}\left\{\Lambda_{j}^{*}\left(H_{j}\right): j \in N\right\}\right)^{\perp}, \forall j \in N .
\end{aligned}
$$

Let $P$ be the orthogonal projection from $H$ onto $\overline{\operatorname{span}}\left\{\Lambda_{j}^{*}\left(H_{j}\right): j \in N\right\}$. Then we have $T x=T P x, \forall x \in H$. So

$$
\|T x\|^{2}=\|T P x\|^{2}=\left\|\sum_{j \in N} \theta_{j}^{*} \Lambda_{j} P x\right\|^{2}=\sum_{j \in N}\left\|\Lambda_{j} P x\right\|^{2} .
$$

Since

$$
a\|P x\|^{2} \leq \sum_{j \in N}\left\|\Lambda_{j} P x\right\|^{2} \leq b\|P x\|^{2}, \forall x \in H
$$

we have that $a\|P x\|^{2} \leq\|T x\|^{2} \leq b\|P x\|^{2}, \forall x \in H$. So $a P \leq T^{*} T \leq b P$.

$\Leftarrow$ : If there exists an operator $T \in B(H)$ such that $\Lambda_{j}=\theta_{j} T, \forall j \in N$ and $a P \leq$ $T^{*} T \leq b P$ for some orthogonal projection $P$, then

$$
\sum_{j \in N}\left\|\Lambda_{j} x\right\|^{2}=\sum_{j \in N}\left\|\theta_{j} T x\right\|^{2}=\|T x\|^{2}
$$

Since for all $x \in P H$, we have that $a\langle P x, x\rangle \leq\left\langle T^{*} T x, x\right\rangle \leq b\langle P x, x\rangle$, i.e., $a\|x\|^{2} \leq$ $\|T x\|^{2} \leq b\|x\|^{2}, \forall x \in P H$, for every $x \in P H$, we have

$$
a\|x\|^{2} \leq \sum_{j \in N}\left\|\Lambda_{j} x\right\|^{2} \leq b\|x\|^{2}
$$


Since $a P \leq T^{*} T \leq b P, a\|P x\|^{2} \leq\|T x\|^{2} \leq b\|P x\|^{2}$. It is easy to see that $\operatorname{Ker} T=$ $\operatorname{Ker} P=\operatorname{Range}(I-P)$. It implies that

$$
\begin{aligned}
& P H=(K e r T)^{\perp}=\overline{T^{*}(H)}=\overline{T^{*} \overline{\operatorname{span}}\left\{\theta_{j}^{*}\left(H_{j}\right): j \in N\right\}} \\
& =\overline{\operatorname{span}}\left\{T^{*} \theta_{j}^{*}\left(H_{j}\right): j \in N\right\}=\overline{\operatorname{span}\left\{\Lambda_{j}^{*}\left(H_{j}\right): j \in N\right\} .}
\end{aligned}
$$

So $\left\{\Lambda_{j}: j \in N\right\}$ is a $g$-frame sequence with bounds $a$ and $b$.

From the arguments of the above theorem, the following result is obvious.

Corollary 3.4. Suppose that $\left\{\theta_{j}: j \in N\right\}$ is a g-orthonormal basis for $H$. Then $\left\{\Lambda_{j}: j \in N\right\}$ is a $g$-frame for $H$ with fame bounds $a$ and $b$ if and only if there exists a unique $T \in B(H)$ such that $\Lambda_{j}=\theta_{j} T, \forall j \in N$ and $a I \leq T T^{*} \leq b I$.

Theorem 3.5. Let $\left\{\Lambda_{j}: j \in N\right\}$ be a g-orthonormal basis for $H$ and $T \in B(H)$. Then $\left\{\Lambda_{j} T: j \in N\right\}$ is a g-orthonormal basis if and only if $T$ is unitary.

Proof. $\quad \Rightarrow$ : Since $\left\{\Lambda_{j}: j \in N\right\}$ is a $g$-orthonormal basis for $H$, we have $\sum_{j \in N}\left\|\Lambda_{j} T f\right\|^{2}=\|T f\|^{2}$, for any $f \in H$. On the other hand, $\left\{\Lambda_{j} T: j \in N\right\}$ is also a $g$-orthonormal basis for $H$, we have $\sum_{j \in N}\left\|\Lambda_{j} T f\right\|^{2}=\|f\|^{2}$ for any $f \in H$. So $\|T f\|^{2}=\|f\|^{2}$, which implies that $T$ is an isometry. Hence $T^{*} T=I$. On the other hand, for any $i, j \in N$ and $g_{i} \in H_{i}, g_{j} \in H_{j}$, we have

$$
\left\langle T T^{*} \Lambda_{j}^{*} g_{j}, \Lambda_{i}^{*} g_{i}\right\rangle=\left\langle T^{*} \Lambda_{j}^{*} g_{j}, T^{*} \Lambda_{i}^{*} g_{i}=\delta_{i, j}\left\langle g_{j}, g_{i}\right\rangle,\right.
$$

since $\left\{\Lambda_{j} T: j \in N\right\}$ is a $g$-orthonormal basis. And

$$
\left\langle\Lambda_{j}^{*} g_{j}, \Lambda_{i}^{*} g_{i}\right\rangle=\delta_{i, j}\left\langle g_{j}, g_{i}\right\rangle,
$$

since $\left\{\Lambda_{j}: j \in N\right\}$ is a $g$-orthonormal basis. So $\left\langle T T^{*} \Lambda_{j}^{*} g_{j}, \Lambda_{i}^{*} g_{i}\right\rangle=\left\langle\Lambda_{j}^{*} g_{j}, \Lambda_{i}^{*} g_{i}\right\rangle$. It implies that $T T^{*} \Lambda_{j}^{*} g_{j}=\Lambda_{j}^{*} g_{j}$ for any $j \in N$ and $g_{j} \in H_{j}$. Since $\left\{\Lambda_{j}: j \in N\right\}$ is a $g$-orthonormal basis, $\left\{\Lambda_{j}: j \in N\right\}$ is a $g$-frame by the definition of $g$-orthonormal basis. So $\left\{\Lambda_{j}: j \in N\right\}$ is $g$-complete by Lemma 2.14. Hence $\overline{\operatorname{span}}\left\{\Lambda_{j}^{*}\left(H_{j}\right)\right\}=H$ by Lemma 2.15. So $T T^{*}=I$. Hence $T$ is unitary.

$\Leftarrow$ : If $T$ is unitary and $\left\{\Lambda_{j}: j \in N\right\}$ is a $g$-orthonormal basis, then for any $i, j \in N$ and $g_{i} \in H_{i}, g_{j} \in H_{j}$, we have

$$
\begin{aligned}
& \left\langle\left(\Lambda_{j} T\right)^{*} g_{j},\left(\Lambda_{i} T\right)^{*} g_{i}\right\rangle=\left\langle T^{*} \Lambda_{j}^{*} g_{j}, T^{*} \Lambda_{i}^{*} g_{i}\right\rangle \\
& =\left\langle T T^{*} \Lambda_{j}^{*} g_{j}, \Lambda_{i}^{*} g_{i}\right\rangle=\left\langle\Lambda_{j}^{*} g_{j}, \Lambda_{i}^{*} g_{i}\right\rangle \\
& =\delta_{i, j}\left\langle g_{j}, g_{i}\right\rangle .
\end{aligned}
$$

And for any $f \in H$, we have

$$
\sum_{j \in N}\left\|\Lambda_{j} T f\right\|^{2}=\|T f\|^{2}=\|f\|^{2} .
$$

Thus, $\left\{\Lambda_{j} T: j \in N\right\}$ is a $g$-orthonormal basis. 
Theorem 3.6. Suppose that $\left\{\Lambda_{j} \in B\left(H, H_{j}\right): j \in N\right\}$ is a g-basis and $T \in$ $B(H)$. If $T$ is invertible, then $\left\{\Lambda_{j} T^{*}: j \in N\right\}$ and $\left\{\Lambda_{j} T: j \in N\right\}$ are g-bases for $H$. Conversely, if $\left\{\Lambda_{j} T^{*}: j \in N\right\}$ and $\left\{\Lambda_{j} T: j \in N\right\}$ are g-bases for $H$ and $T$ has closed range, then $T$ is invertible.

Proof. Since $T$ is invertible, there exists an operator $T^{-1} \in B(H)$ such that $T T^{-1}=I$. Since $\left\{\Lambda_{j}: j \in N\right\}$ is a $g$-basis for $H$, for any $f \in H$, there exists a unique sequence $\left\{g_{j}: j \in N\right\}$ such that $T^{-1} f=\sum_{j \in N} \Lambda_{j}^{*} g_{j}$. So $T T^{-1} f=\sum_{j \in N} T \Lambda_{j}^{*} g_{j}$, i.e., $f=\sum_{j \in N}\left(\Lambda_{j} T^{*}\right)^{*} g_{j}$. Suppose that there is another sequence $\left\{h_{j}: j \in N\right\}$ such that $f=\sum_{j \in N}\left(\Lambda_{j} T^{*}\right)^{*} h_{j}$. Then $\sum_{j \in N}\left(\Lambda_{j} T^{*}\right)^{*}\left(g_{j}-h_{j}\right)=0$, i.e., $\sum_{j \in N} T \Lambda_{j}^{*}\left(g_{j}-\right.$ $\left.h_{j}\right)=0$. Hence, $T^{-1} \sum_{j \in N} T \Lambda_{j}^{*}\left(g_{j}-h_{j}\right)=0$. So $\sum_{j \in N} \Lambda_{j}^{*}\left(g_{j}-h_{j}\right)=0$. Since $\left\{\Lambda_{j}: j \in N\right\}$ is a $g$-basis, $g_{j}-h_{j}=0$ for every $j \in N$, i.e., $g_{j}=h_{j}$ for every $j \in N$. So $\left\{\Lambda_{j} T^{*}: j \in N\right\}$ is a $g$-basis for $H$. If $T$ is invertible, then $T^{*}$ is also invertible. So $\left\{\Lambda_{j} T: j \in N\right\}$ is a $g$-basis for $H$ as well.

Conversely, assume $\left\{\Lambda_{j} T^{*}: j \in N\right\}$ and $\left\{\Lambda_{j} T: j \in N\right\}$ are $g$-bases for $H$ and $T$ has close range. To prove that $T$ is invertible, it is sufficient to prove that $\operatorname{Ker} T=\{0\}$ and Range $(T)=H$. Suppose that $f \in H$ and $T f=0$. Then there exists a sequence $\left\{g_{j}: j \in N\right\}$ such that $f=\sum_{j \in N} \Lambda_{j}^{*} g_{j}$. So $T f=\sum_{j \in N} T \Lambda_{j}^{*} g_{j}=$ $\sum_{j \in N}\left(\Lambda_{j} T^{*}\right)^{*} g_{j}$. Hence $\sum_{j \in N}\left(\Lambda_{j} T^{*}\right)^{*} g_{j}=0$. So $g_{j}=0, \forall j \in N$, which implies that $f=0$. So $\operatorname{Ker} T=\{0\}$. Similarly, $\operatorname{Ker} T^{*}=\{0\}$. Since $\operatorname{Range}(T)=$ $\overline{\operatorname{Range}}(T)=\left(\operatorname{Ker} T^{*}\right)^{\perp}=H, T$ is invertible.

Theorem 3.7. Let $\left\{\Lambda_{j}: j \in N\right\}$ be a g-Riesz basis for $H,\left\{\widetilde{\Lambda}_{j}: j \in N\right\}$ be its dual $g$-Riesz basis and $T \in B(H)$. Then $\left\{\Lambda_{j} T: j \in N\right\}$ is a $g$-Riesz basis if and only if $T$ is invertible. Moreover the dual g-Riesz basis of $\left\{\Lambda_{j} T: j \in N\right\}$ is $\left\{\widetilde{\Lambda}_{j}\left(T^{*}\right)^{-1}: j \in N\right\}$.

Proof. Since $\left\{\Lambda_{j}: j \in N\right\}$ is a $g$-Riesz basis for $H$, there exists a $g$-orthonormal basis $\left\{\theta_{j}: j \in N\right\}$ and an invertible operator $U \in B(H)$ such that $\Lambda_{j}=\theta_{j} U, j \in N$ by Lemma 2.16. So $\Lambda_{j} T=\theta_{j} U T, j \in N$. Since $U$ is invertible, $U T$ is invertible if and only if $T$ is invertible. It implies that $\left\{\Lambda_{j} T: j \in N\right\}$ is a $g$-Riesz basis if and only if $T$ is invertible by Lemma 2.16. Suppose that the frame operator associated with $\left\{\Lambda_{j}: j \in N\right\}$ and $\left\{\Lambda_{j} T: j \in N\right\}$ are $S$ and $S^{\prime}$ respectively. Then $S f=\sum_{j \in N} \Lambda_{j}^{*} \Lambda_{j} f$ and $S^{\prime} f=\sum_{j \in N} T^{*} \Lambda_{j}^{*} \Lambda_{j} T f$. So $S^{\prime}=T^{*} S T$. Since

$$
\Lambda_{j} T\left(S^{\prime}\right)^{-1}=\Lambda_{j} T T^{-1} S^{-1}\left(T^{*}\right)^{-1}=\Lambda_{j} S^{-1}\left(T^{*}\right)^{-1}=\widetilde{\Lambda}_{j}\left(T^{*}\right)^{-1} .
$$

Hence the dual $g$-Riesz basis of $\left\{\Lambda_{j} T: j \in N\right\}$ is $\left\{\widetilde{\Lambda}_{j}\left(T^{*}\right)^{-1}: j \in N\right\}$.

\section{General Operator Parameterizations of $g$-Frames}

In this section, we mainly parameterize the $g$-frames in general without assuming the existence of $g$-orthonormal bases. All these results are inspired by the results in [24]. 
Theorem 4.1. Let $\left\{\Lambda_{j}: j \in N\right\}$ be a $g$-frame of $H$ with frame bounds $0<A<B$ and $T \in B(H)$. Then $\left\{\Lambda_{j} T: j \in N\right\}$ is a $g$-frame of $H$ if and only if there exists a positive constant $\gamma$ such that $\|T f\|^{2} \geq \gamma\|f\|^{2}$.

Proof. $\Leftarrow$ : Since $\left\{\Lambda_{j}: j \in N\right\}$ is a $g$-frame with bounds $0<A<B$,

$$
\begin{aligned}
& \sum_{j \in N}\left\|\Lambda_{j} T f\right\|^{2} \leq B\|T f\|^{2} \leq B \cdot\|T\|^{2}\|f\|^{2}, \text { and } \\
& \sum_{j \in N}\left\|\Lambda_{j} T f\right\|^{2} \geq A\|T f\|^{2} \geq A \cdot \gamma\|f\|^{2} .
\end{aligned}
$$

So, $\left\{\Lambda_{j} T: j \in N\right\}$ is a $g$-frame of $H$ with frame bounds $A \gamma$ and $B\|T\|^{2}$.

$\Rightarrow$ : Suppose $\left\{\Lambda_{j} T: j \in N\right\}$ is a $g$-frame with frame bounds $a$ and $b$. Then for any $f \in H$

$$
a\|f\|^{2} \leq \sum_{j \in N}\left\|\Lambda_{j} T f\right\|^{2} \leq B\|T f\|^{2} .
$$

So $\|T f\|^{2} \geq \frac{a}{B}\|f\|^{2}$, for any $f \in H$. Let $\gamma=\frac{a}{B}$, then the proof is completed.

Similarly, we have the following results. We leave the details for the readers.

Corollary 4.2. Let $M$ be a closed subspace of $H$ and $\left\{\Lambda_{j}: j \in N\right\}$ be a $g$-frame of $H$. $T$ is a bounded linear operator from $H$ into $M$. Then $\left\{\Lambda_{j} T^{*}: j \in N\right\}$ is a $g$-frame of $M$ if and only if there exists a positive constant $\gamma$ such that

$$
\left\|T^{*} f\right\|^{2} \geq \gamma\|f\|^{2}, \forall f \in M
$$

Corollary 4.3. Let $\left\{\Lambda_{j}: j \in N\right\}$ be a g-tight frame of $H$ with frame bound $B$ and $T \in B(H)$. Then $\left\{\Lambda_{j} T: j \in N\right\}$ is a g-tight frame of $H$ with frame bound $b$ if and only if $\|T f\|^{2}=\frac{b}{B}\|f\|^{2}$.

Theorem 4.4. Let $M$ be a closed subspace of $H$ and $\left\{\Lambda_{j}: j \in N\right\}$ be a g-frame of $H$ with frame bounds $A$ and $B$. Suppose $P$ is the orthogonal projection from $H$ onto $M,\left\{\widetilde{\Lambda}_{j}: j \in N\right\}$ is the canonical dual g-frame of $\left\{\Lambda_{j}: j \in N\right\}$. Then $\left\{\widetilde{\Lambda}_{j} P\right\}$ is a dual g-frame of $\left\{\Lambda_{j} P: j \in N\right\}$ in M. Moreover, $A$ and $B$ are also frame bounds of $\left\{\Lambda_{j} P: j \in N\right\}$.

Proof. Since $P$ is the orthogonal projection from $H$ onto $M$, we have that $P$ $=P^{*}$ and $\|P f\|^{2}=\|f\|^{2}$ for any $f \in M$. It follows that $\left\{\Lambda_{j} P: j \in N\right\}$ and $\left\{\widetilde{\Lambda}_{j} P: j \in N\right\}$ are $g$-frames of $M$ by Theorem 4.1. Since for every $f \in M$, we have

$$
f=\sum_{j \in N} \widetilde{\Lambda}_{j}^{*} \Lambda_{j} f=\sum_{j \in N} \widetilde{\Lambda}_{j}^{*} \Lambda_{j} P f .
$$


Since $f=P f$ for every $f \in M$, we have

$$
\begin{aligned}
& f=P f=P \sum_{j \in N} \widetilde{\Lambda}_{j}^{*} \Lambda_{j} P f \\
& =\sum_{j \in N} P \widetilde{\Lambda}_{j}^{*} \Lambda_{j} P f=\sum_{j \in N}\left(\widetilde{\Lambda}_{j} P\right)^{*}\left(\Lambda_{j} P\right) f .
\end{aligned}
$$

So $\left\{\widetilde{\Lambda}_{j} P: j \in N\right\}$ is a dual $g$-frame of $\left\{\Lambda_{j} P: j \in N\right\}$ in $M$. Since for any $f \in M$

$$
\sum_{j \in N}\left\|\Lambda_{j} P f\right\|^{2}=\sum_{j \in N}\left\|\Lambda_{j} f\right\|^{2}
$$

$A$ and $B$ are also frame bounds of $\left\{\Lambda_{j} P: j \in N\right\}$.

5. Operator Parameterizations of $g$-Frames with Special Structures

In this section, some special $g$-frames are parameterized in terms of operators.

Given a Hilbert space $K$, we define sequence space $l^{2}(K)$ and $l^{\infty}(K)$ by

$$
l^{2}(K)=\left\{\left\{f_{i}\right\} \mid f_{i} \in K, \sum_{i \in N}\left\|f_{i}\right\|^{2}<\infty\right\},
$$

with the inner product defined by $\left\langle\left\{f_{i}\right\},\left\{g_{i}\right\}\right\rangle=\sum_{i \in N}\left\langle f_{i}, g_{i}\right\rangle$. It is easy to see that $l^{2}(K)$ is a Hilbert space.

$$
l^{\infty}(K)=\left\{\left\{f_{i}\right\} \mid f_{i} \in K, \text { and } \exists C>0 \text { such that }\left\|f_{i}\right\| \leq C\right\},
$$

with the norm defined as $\left\|\left\{f_{i}\right\}\right\|=\sup _{i \in N}\left\|f_{i}\right\|, l^{\infty}(K)$ is a Banach space. Let $U=\left(u_{m, n}\right)$ be a bounded linear operator on $l^{2}(N)$. Since

$$
\begin{aligned}
& \left\|\sum_{j \in N} u_{j, n} g_{j}\right\|^{2} \leq\left(\sum_{j \in N}\left|u_{j, n}\right|\left\|g_{j}\right\|\right)^{2} \\
\leq & \sum_{j \in N}\left|u_{j, n}\right|^{2} \cdot \sum_{j \in N}\left\|g_{j}\right\|^{2}=\left\|U e_{n}\right\|^{2}\left\|\left\{g_{i}\right\}\right\|^{2} \\
\leq & \|U\|^{2} \cdot\left\|\left\{g_{i}\right\}\right\|^{2},
\end{aligned}
$$

where $\left\{e_{n}: n \in N\right\}$ denotes the standard orthonormal basis of $l^{2}(N)$, and $\left\{g_{j}\right\} \in$ $l^{2}\left(\oplus H_{j}\right)$. So $\sum_{j \in N} u_{j, n} g_{j}$ is convergent. So $U$ induces a linear bounded operator $T_{U}: l^{2}\left(\oplus H_{j}\right) \rightarrow l^{\infty}(K)$ by

$$
\left(T_{U}\left\{g_{i}\right\}\right)_{n}=\sum_{j \in N} u_{j, n} g_{j}
$$


and $\left\|T_{U}\right\| \leq\|U\|$. Particularly, if $M=\sum_{i, j \in N}\left|u_{i, j}\right|^{2}<\infty$, then

$$
\begin{aligned}
& \sum_{n \in N}\left\|\left(T_{U}\left\{g_{i}\right\}\right)_{n}\right\|^{2}=\sum_{n \in N}\left\|\sum_{j \in N} u_{j, n} g_{j}\right\|^{2} \\
\leq & \sum_{n \in N}\left(\sum_{j \in N}\left|u_{j, n}\right|\left\|g_{j}\right\|\right)^{2} \leq \sum_{n \in N} \sum_{j \in N}\left|u_{j, n}\right|^{2} \sum_{j \in N}\left\|g_{j}\right\|^{2} \\
= & M \sum_{j \in N}\left\|g_{j}\right\|^{2} .
\end{aligned}
$$

So, $T_{U}$ is a linear bounded operator from $l^{2}\left(\oplus H_{i}\right)$ into $l^{2}(K)$ with $\left\|T_{U}\right\| \leq \sum_{i, j \in N}\left|u_{i, j}\right|^{2}$. From the above analysis, it is easy to get the following result.

Theorem 5.1. Let $\left\{\Lambda_{j}: j \in N\right\}$ be a g-frame of $H$ with frame bounds $A$ and $B$, and let $U=\left(u_{i, j}\right)$ be a linear bounded operator on $l^{2}(N)$ with $M=\sum_{i, j \in N}\left|u_{i, j}\right|^{2}<$ $\infty$. Let $\Gamma_{n} \in B(H, K), n \in N$ be defined by $\Gamma_{n} f=\left(T_{U}\left\{\Lambda_{i} f\right\}\right)_{n}=\sum_{j \in N} u_{n, j} \Lambda_{j} f$. Then $\left\{\Gamma_{n}: n \in N\right\}$ is a $g$-Bessel sequence of $H$ with respect to $K$ with bound $M B$.

Theorem 5.2. Let $\left\{\Lambda_{j}: j \in N\right\}$ be a g-frame of $H$ with frame bounds $A$ and $B$ and $U$ be a linear bounded operator on $l^{2}(N)$ such that $T_{U}$ is a linear bounded operator from $l^{2}\left(\oplus H_{i}\right)$ into $l^{2}(V) . \Gamma_{n} f=\left(T_{U}\left\{\Lambda_{i} f\right\}\right)_{n}=\sum_{j \in N} u_{n, j} \Lambda_{j} f$ for each $n \in N$. Then $\left\{\Gamma_{n}: n \in N\right\}$ is a $g$-frame of $H$ with respect to $V$ if and only if there exists a positive constant $\gamma$ such that for all $x \in X=\operatorname{Range}(T)$, where $T$ is the analysis operator associated with $\left\{\Lambda_{j}: j \in N\right\}$, we have

$$
\left\|T_{U} x\right\|^{2} \geq \gamma\|x\|^{2} .
$$

Proof. $\Rightarrow$ : Since $\left\{\Lambda_{j}: j \in N\right\}$ be a $g$-frame of $H$ with frame bounds $A$ and $B$, $A\|f\|^{2} \leq \sum_{j \in N}\left\|\Lambda_{j} f\right\|^{2} \leq B\|f\|^{2}, \forall f \in H$. So $\left\{\Lambda_{j} f: j \in N\right\} \in l^{2}\left(\oplus H_{j}\right)$ and

$$
\begin{aligned}
& \sum_{j \in N}\left\|\Gamma_{j} f\right\|^{2}=\sum_{j \in N}\left\|\left(T_{U}\left\{\Lambda_{i} f\right\}\right)_{j}\right\|^{2}=\left\|T_{U}\left\{\Lambda_{i} f\right\}\right\|^{2} \\
& \leq\left\|T_{U}\right\|^{2}\left\|\left\{\Lambda_{i} f\right\}\right\|^{2}=\left\|T_{U}\right\|^{2} \cdot \sum_{i \in N}\left\|\Lambda_{i} f\right\|^{2} \leq\left\|T_{U}\right\|^{2} \cdot B \cdot\|f\|^{2} .
\end{aligned}
$$

On the other hand, since $\left\{\Lambda_{j} f: j \in N\right\} \in X=\operatorname{Range}(T)$, we have

$$
\sum_{j \in N}\left\|\Gamma_{j} f\right\|^{2}=\left\|T_{U}\left\{\Lambda_{i} f\right\}\right\|^{2} \geq \gamma\left\|\left\{\Lambda_{j} f\right\}\right\|^{2} \geq \gamma \cdot A \cdot\|f\|^{2} .
$$

Thus, $\left\{\Gamma_{j}: j \in N\right\}$ is a $g$-frame of $H$ with respect to $V$.

$\Rightarrow$ : Assume that $\left\{\Gamma_{j}: j \in N\right\}$ is a $g$-frame of $H$ with frame bounds $a$ and $b$ with respect to $V$. Then 


$$
b\|f\|^{2} \geq \sum_{j \in N}\left\|\Gamma_{j} f\right\|^{2}=\left\|T_{U}\left\{\Lambda_{i} f\right\}\right\|^{2} \geq a\|f\|^{2} .
$$

On the other hand, since $\left\{\Lambda_{j}: j \in N\right\}$ is a $g$-fame of $H$ with frame bounds $A$ and $B$, we have

$$
B\|f\|^{2} \geq \sum_{j \in N}\left\|\Lambda_{j} f\right\|^{2} \geq A\|f\|^{2}
$$

So,

$$
\left\|T_{U}\left\{\Lambda_{i} f\right\}\right\|^{2} \geq a\|f\|^{2} \geq \frac{a}{B} \cdot \sum_{j \in N}\left\|\Lambda_{j} f\right\|^{2}=\frac{a}{B} \cdot\left\|\left\{\Lambda_{i} f\right\}\right\|^{2} .
$$

The proof is completed.

Theorem 5.3. Suppose $\operatorname{dim} H_{i}<\infty$ for any $i \in N$. Then $\left\{\Lambda_{i} \in B\left(H, H_{i}\right)\right.$ : $i \in N\}$ is a g-frame of $H$ if and only if there exists a bounded linear operator $Q$ from $l^{2}\left(\oplus H_{i}\right)$ onto $H$ such that $Q\left(\left\{\delta_{n, j} e_{n, k_{n}}\right\}_{n=1}^{\infty}\right)=\Lambda_{j}^{*} e_{j, k_{j}}$ for each $j \in N$, where $\left\{e_{n, k_{n}}: k_{n}=1,2, \cdots, K_{n}\right\}$ denotes an orthonormal basis of $H_{n}$.

Proof. Let $F_{j, k_{j}}=e_{n, k_{n}}$ if $j=n, F_{j, k_{j}}=0$, if $j \neq n$. Then $\left\{F_{j, k_{j}}: j \in N, k_{j}=\right.$ $\left.1,2, \cdots, K_{j}\right\}=\left\{\left\{\delta_{n, j} e_{n, k_{n}}\right\}_{n=1}^{\infty}: j \in N, k_{n}=1,2, \cdots, K_{n}\right\}$ is an orthonormal basis of $l^{2}\left(\oplus H_{j}\right)$.

$\Rightarrow$ : If $\left\{\Lambda_{i} \in B\left(H, H_{i}\right): i \in N\right\}$ is a $g$-frame for $H$ with respect to $\left\{H_{i}: i \in N\right\}$, then there exist positive constants $A$ and $B$ such that for any $f \in H, A\|f\|^{2} \leq$ $\sum_{i \in N}\left\|\Lambda_{i} f\right\|^{2} \leq B\|f\|^{2}$. In particular, $\left\{\Lambda_{i} f: i \in N\right\} \in l^{2}\left(\oplus H_{i}\right)$. Let $U$ be the analysis operator associated with $\left\{\Lambda_{i}: i \in N\right\}$ and $Q=U^{*}$ be the synthesis operator associated with $\left\{\Lambda_{i}: i \in N\right\}$. Then $Q$ maps $l^{2}\left(\oplus H_{i}\right)$ onto $H$ and for any $\left\{g_{i}: i \in N\right\} \in l^{2}\left(\oplus H_{i}\right), Q\left\{g_{i}\right\}=\sum_{i \in N} \Lambda_{i}^{*} g_{i}$ by Lemma 2.12. Since for all $f \in H, j \in N$, we have

$$
\begin{gathered}
\left\langle f, Q F_{j, k_{j}}\right\rangle=\left\langle Q^{*} f, F_{j, k_{j}}\right\rangle=\left\langle U f, F_{j, k_{j}}\right\rangle \\
=\left\langle\left\{\Lambda_{i} f\right\}, F_{j, k_{j}}\right\rangle=\left\langle\Lambda_{j} f, e_{j, k_{j}}\right\rangle=\left\langle f, \Lambda_{j}^{*} e_{j, k_{j}}\right\rangle,
\end{gathered}
$$

it follows that $Q F_{j, k_{j}}=\Lambda_{j}^{*} e_{j, k_{j}}$, for all $j \in N$.

$\Leftarrow$ : Suppose that $Q$ is a bounded linear operator from $l^{2}\left(\oplus H_{j}\right)$ onto $H$ such that $Q F_{j, k_{j}}=\Lambda_{j}^{*} e_{j, k_{j}}$, for all $j \in N$. Since $\left\{F_{j, k_{j}}: k_{j}=1,2, \cdots, K_{j}, j \in N\right\}$ is an orthonormal basis for $l^{2}\left(\oplus H_{j}\right)$, then for any $f \in H$, we have

$$
\begin{aligned}
& Q^{*}(f)=\sum_{j \in N} \sum_{k_{j}=1}^{K_{j}}\left\langle Q^{*}(f), F_{j, k_{j}}\right\rangle F_{j, k_{j}}=\sum_{j \in N} \sum_{k_{j}=1}^{K_{j}}\left\langle f, Q F_{j, k_{j}}\right\rangle F_{j, k_{j}} \\
= & \sum_{j \in N} \sum_{k_{j}=1}^{K_{j}}\left\langle f, \Lambda_{j}^{*} e_{j, k_{j}}\right\rangle F_{j, k_{j}}=\sum_{j \in N} \sum_{k_{j}=1}^{K_{j}}\left\langle\Lambda_{j} f, e_{j, k_{j}}\right\rangle F_{j, k_{j}} .
\end{aligned}
$$


Hence

$$
\left\|Q^{*} f\right\|^{2}=\sum_{j \in N} \sum_{k_{j}=1}^{K_{j}}\left|\left\langle\Lambda_{j} f, e_{j, k_{j}}\right\rangle\right|^{2}=\sum_{j \in N}\left\|\Lambda_{j} f\right\|^{2} .
$$

Since $Q$ is onto, there exists a bounded linear operator $Q^{\dagger}$ such that $Q Q^{\dagger}=I_{H}$ by Lemma 2.9. It implies that $\left(Q^{\dagger}\right)^{*} Q^{*}=I_{H}$. So for any $f \in H,\left\|\left(Q^{\dagger}\right)^{*} Q^{*} f\right\|=\|f\|$. It implies that

$$
\|f\| \leq\left\|\left(Q^{\dagger}\right)^{*}\right\| \cdot\left\|Q^{*} f\right\|, \text { i.e., }\left\|Q^{*} f\right\| \geq \frac{1}{\left\|\left(Q^{\dagger}\right)^{*}\right\|} \cdot\|f\| .
$$

So

$$
\frac{1}{\left\|\left(Q^{\dagger}\right)^{*}\right\|^{2}} \cdot\|f\|^{2} \leq \sum_{j \in N}\left\|\Lambda_{j} f\right\|^{2}=\left\|Q^{*} f\right\|^{2} \leq\left\|Q^{*}\right\|^{2} \cdot\|f\|^{2}
$$

It follows that $\left\{\Lambda_{j}: j \in N\right\}$ is a $g$-frame of $H$ with respect to $\left\{H_{i}: i \in N\right\}$.

Theorem 5.4. If $\left\{H_{j}: j \in N\right\}$ is a sequence of closed subspaces of Hilbert space $V$ with $\operatorname{dim}_{j}<\infty$ for each $j \in N$, then every g-frame of $H$ with respect to $\left\{H_{j}: j \in N\right\}$ is equivalent to one of the $g$-frames $\left\{J_{j}^{*} P: j \in N\right\}$, where $P$ is some orthogonal projection on $l^{2}\left(\oplus H_{j}\right)$ and $J_{i}$ is the natural embedding of $H_{j}$ into $l^{2}\left(\oplus H_{j}\right)$, which is defined by $J_{j}\left(g_{j}\right)=\left\{\delta_{i, j} g_{j}: i \in N\right\}$.

Proof. If $P$ is some orthogonal projection of $l^{2}\left(\oplus H_{j}\right)$ onto a closed subspace $X$ of $l^{2}\left(\oplus H_{j}\right)$, then

$$
\left(J_{j}^{*} P\right)^{*} e_{j, k_{j}}=P\left(J_{j} e_{j, k_{j}}\right)=P\left(\left\{\delta_{n, j} e_{j, k_{j}}: n \in N\right\}\right),
$$

where $\left\{e_{j, k_{j}}: k_{j}=1,2, \cdots, K_{j}\right\}$ is an orthonormal basis for $H_{j}, \forall j \in N$. So $\left\{J_{j}^{*} P: j \in N\right\}$ is a $g$-frame for $X$ with respect to $\left\{H_{j}: j \in N\right\}$ by Theorem 5.3. Now, suppose that $\left\{\Lambda_{j}: j \in N\right\}$ is any $g$-frame for $H$ with respect to $\left\{H_{j}: j \in N\right\}$. Then by Theorem 5.3, there exists a bounded surjective linear operator $Q: l^{2}\left(\oplus H_{j}\right) \rightarrow H$ such that for each $n \in N$, we have

$$
Q\left(\left\{\delta_{n, j} e_{n, k_{n}}\right\}_{j=1}^{\infty}\right)=\Lambda_{n}^{*} e_{n, k_{n}} .
$$

But $\left\{\delta_{n, j} e_{n, k_{n}}\right\}=J_{n}\left(e_{n, k_{n}}\right)$, so $Q J_{n}\left(e_{n, k_{n}}\right)=\Lambda_{n}^{*} e_{n, k_{n}}$ for each $n \in N$. Let $P$ be the orthogonal projection of $l^{2}\left(\oplus H_{j}\right)$ onto $(\operatorname{Ker} Q)^{\perp}$. Then

$$
\begin{aligned}
& \Lambda_{n}^{*} e_{n, k_{n}}=Q\left(\left\{\delta_{n, j} e_{n, k_{n}}\right\}_{j=1}^{\infty}\right) \\
= & Q P\left(\left\{\delta_{n, j} e_{n, k_{n}}\right\}_{j=1}^{\infty}\right)=Q P J_{n}\left(e_{n, k_{n}}\right), \forall n \in N .
\end{aligned}
$$

So, $\Lambda_{n}^{*}=Q P J_{n}, \forall n \in N$. It follows that $\Lambda_{n}=\left(P J_{n}\right)^{*} Q^{*}=\left(J_{n}^{*} P\right) Q^{*}, \forall n \in N$. Since $Q^{*}$ is an isomorphism from $H$ onto $(\operatorname{Ker} Q)^{\perp},\left\{\Lambda_{j}: j \in N\right\}$ is equivalent to $\left\{J_{j}^{*} P: j \in N\right\}$. 


\section{REFERENCES}

1. D. Gabor, Theory of communications, Jour. Inst. Elec. Eng., 93 (1946), 429-457.

2. R. J. Duffin and A. C. Schaeffer, A class of nonharmonic Fourier series, Transactions of AMS, 72, 341-366.

3. I. Daubechies, A. Grossmann and Y. Meyer, Painless nonorthognal expansions, J. Math. Phys., 27 (1986), 1271-1283.

4. D. Han and D. R. Larson, Frames, basis and group representations, Mem. Amer. Math. Soc., 147 (2000).

5. O. Christensen, An Introduction to Frames and Riestz Bases, Birkhauser, Boston, 2003.

6. I. Daubchies, Ten Lectures on Wavelets, SIAM, Philadelphia, 1992.

7. C. Heil and D. Walnut, Continuous and discrete wavelet transforms, SIAM Rev., 31 (1989), 628-666.

8. H. G. Feichtinger and T. Strohmer, Gabor Analysis and Algorithms: Theory and Applications, Birkhauser, Boston, MA, 1998.

9. M. Frazier and B. Jawerth, Decompositions of Besov spaces, Iniana Univ. Math. J., 34 (1985), 777-799.

10. K. Grochenig, Describing functions: Atomic decompositions versus frames, Monatshefte fur Math., 112 (1991), 1-41.

11. Wenchang Sun, $g$-frames and $g$-Riesz bases, J. Math. Anal. Appl., 322 (2006), 437-452.

12. Yu Can Zhu, Characterizations of $g$-Frames and $g$-Riesz Bases in Hilbert Spaces, Acta Mathematica Sinica, 24 (2008), 1727-1736.

13. Abbas Najati, M. H. Faroughi and Asghar Rahimi, $g$-Frames and stability of $g$-frames in Hilbert spaces, Methods of Functional Analysis and Topology, 14(3) (2008), 271-286.

14. Yan Jin Wang and Yu Can Zhu, $g$-Frames and $g$-Frames Sequences in Hilbert Spaces, Acta Mathematica Sinica, 25(12) (2009), 2093-2106.

15. Amir Khosravi and kamran Musazadeh, Fusion frames and $g$-frames, J. Math. Anal. Appl., 342 (2008), 1068-1083.

16. Ming Ling Ding and Yu Can Zhu, $g$-Besselian Frames in Hilbert Spaces, Acta Mathematica, 26 (2010), 2117-2130.

17. Abdolaziz Abdollahi and Elham Rahimi, Some results on $g$-frames in Hilbert spaces, Turk J. Math., 35 (2011), 695-704.

18. M. R. Abdollahpour and A. Najati, Besselian $g$-frames and $g$-Riesz bases, Appl. Anal. Discrete Math., 5 (2011), 259-270.

19. Jian Zhen Li and Yu can Zhu, Exact $g$-frames in Hilbert spaces, J. Math. Anal. Appl., 374 (2011), 201-209. 
20. Xunxiang Guo, g-Bases in Hilbert spaces, Abstract and Applied Analysis, Vol. 2012, Article ID 923729, 14 pages, doi:10.1155/2012/923729.

21. Jameson Cahill, Peter G. Casazza and Gitta Kutyniok, Operators and frames, preprint.

22. P. G. Casazza and G. Kutyniok, A generalization of Gram-Schmidt orthogonalization generating all Parseval frames, Adv. Comput. Math., 27 (2007), 65-78.

23. Mariano A. Ruiz and Demetrio Stojanoff, Some properties of frames of subspaces obtained by operator theory methods, J. Math. Anal. Appl., 343 (2008), 366-378.

24. Akram Aldroubi, Portraits of frames, Proc. of AMS, 123(6) (1995), 1661-1667.

25. James R. Holub, Pre-frame operators, Besselian frames, and near-Riesz bases in Hilbert spaces, Proc. of AMS, 122(3) (1994), 779-785.

Xunxiang Guo

Department of Mathematics

Southwestern University of Finance and Economics

Chengdu 611130

P. R. China

E-mail: guoxunxiang@yahoo.com 